\title{
Experimental study on a compact methanol-fueled reformer with heat regeneration using ceramic honeycomb (third report: observation of reaction structure and its effect on reforming characteristics)
}

\author{
Y. Rai ${ }^{1}$, H. Kogame ${ }^{1}$, K. Tatsumi ${ }^{1,2}$ \& K. Nakabe ${ }^{1,2}$ \\ ${ }^{1}$ Department of Mechanical Engineering and Science, \\ Kyoto University, Japan \\ ${ }^{2}$ Advanced Research Institute of Fluid Science and Engineering, \\ Kyoto University, Japan
}

\begin{abstract}
A ceramic honeycomb is applied to a 1-kW class compact tubular-type fuel reformer based on non-catalytic partial oxidation (POX) of methanol. Liquid methanol was used due to its easy handling characteristics. It is confirmed that POX in a super-rich condition can be sustained within the reactor. In most conditions, the reaction was stabilized near the outlet surface of the ceramic honeycomb where the maximum temperature is observed. The maximum temperature reaches approximately the adiabatic flame temperature, therefore, the ceramic honeycomb works as an adiabatic layer and a reaction stabilizer which can sustain the reaction at a certain location. The location of the reaction varies with regard to the thermal load conditions. Stable and high conversion rate was obtained when the reaction is stabilized on the outlet surface of the ceramic honeycomb. This robust feature of reaction stabilization is a significant characteristic of the ceramic honeycomb. The reaction characteristic and its effect on the reforming performance are investigated in this study using detailed measurements of temperature distributions and gas components.
\end{abstract}

Keywords: fuel reformer, methanol, non-catalytic partial oxidation, heat regeneration, porous material. 


\section{Introduction}

Due to the recent serious environmental crisis and fossil fuel depletion, hydrogen as an energy carrier has gained more attention as a key to alternative energy supplying system. Hydrogen can be used as a fuel for highly-efficient devices such as fuel cells and hydrogen engines, which emit only water, provided that pure hydrogen is introduced. Moreover, in terms of energy security, hydrogen is a candidate fuel for a secure future energy system since it can be derived from a variety of fuels. However, hydrogen must be artificially synthesized from other materials. Furthermore, due to its low energy density, its highly-efficient delivery and storage systems need technological innovations, which still require considerable time and cost. Therefore, at the transition stage to the future hydrogen society, using conventional infrastructure to deliver hydrocarbon fuel and reforming it at the site of use is the only way to build a highly-efficient hydrogen energy system. The primary motivation of the present study is to construct a compact and simple fuel reformer with a power output of about $1 \mathrm{~kW}$.

Methanol was chosen as the reforming agent in the present study, due to its high energy density and wide availability. Methanol is widely available in the industry and can be synthesized from a variety of hydrocarbon fuels including the abundant coal resources. In addition, methanol can also be derived from biomass materials, yet it need not conflict with the food supply because it can be synthesized from sources such as wood chips or animal wastes [1, 2].

To construct a simple and compact reforming system, partial oxidation (POX; also known as fuel-rich combustion) was chosen. POX is an exothermic reaction that allows a thermally self-sustainable system without a catalyst, which contributes to a compact and low-cost system. Moreover, POX can work with various fuels including low quality fuels, or heavy fuels.

However, due to its fuel-rich condition, flame temperature and heat release rate are markedly lower than for complete oxidation reaction, which prevents a stable reaction with a high reaction rate from being sustained within the reactor. Porous material (e.g. ceramic honeycomb) is often introduced in the reactor to stabilize flame by its flow straightening and highly convective nature. The flame stabilizing effect of porous material is well discussed in terms of the flame location, temperature, and exhaust characteristics [3-5]. In this study, therefore, the role and its effectiveness of ceramic honeycomb to methanol POX will be discussed based on the various thermal load conditions.

Furthermore, heat regeneration effect of ceramic honeycomb will be added to the present reformer by placing the secondary honeycomb in the downstream of the reaction region. Echigo [6] proposed the concept of the radiation converter wherein the enthalpy of the working gas is efficiently converted into radiation emission through highly convective heat transfer between the gas and the solid surface. Okuyama et al. [7] also reported that by adding a porous material, which works as a radiation converter and regenerates the energy from the exhaust gas to the unburned mixture, it was possible to stabilize a super-rich flame beyond the 
flammability limit. This concept of porous radiation converter has been applied to the present reformer to enhance the reforming reaction.

In our study, a compact methanol fuel reformer based on non-catalytic POX has been investigated in terms of equivalence ratio and the configuration of ceramic honeycomb within the reactor $[8,9]$. Then the optimum experimental condition was obtained in a slight fuel rich condition rather than the stoichiometric condition of POX. The increase of methanol conversion was observed by inserting the secondary honeycomb. However, the specific role and its thermal effect were not clearly explained in the previous reports. Therefore, the thermal effect of a ceramic honeycomb on the reforming reaction will be discussed in the present report with regard to the exhaust gas component and reformer performance, correlating the flame stability and its location. We then apply the radiation converter to the present reformer and discussed the energy regeneration effect by the secondary honeycomb.

\section{Experimental setup and procedure}

\subsection{Fuel reformer}

Figure 1(a) shows the schematic view of the present compact fuel reformer. The reformer was composed of the evaporator and the reactor. The evaporator was in the upstream part and was made of a steel pipe with an inner diameter and a length of 28 and $200 \mathrm{~mm}$, respectively. The evaporator was wrapped in two places with electric band heaters for fuel evaporation. Methanol as a fuel was injected through the injector mounted on the surface of the evaporator. The electric valve of the injector was driven by the rectangular signal generated by the function generator for the desired flow rate. Air, on the other hand, was supplied from the air port located at the upstream end of the evaporator. Air flow rate was controlled by a mass flow controller. Electric band heaters were

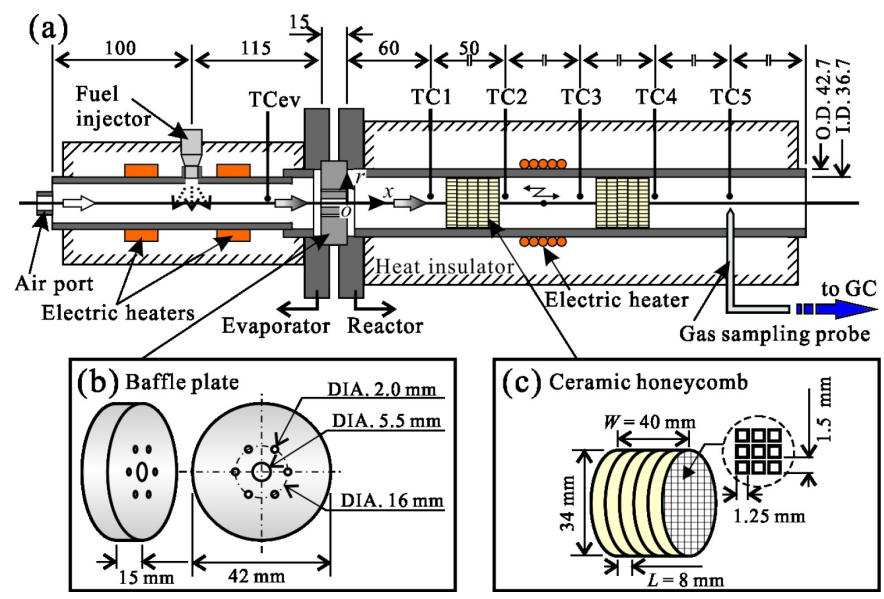

Figure 1: $\quad$ Fuel reformer. 
wrapped around the evaporator and the temperature inside the evaporator was set to $427 \mathrm{~K}$ by adjusting the power supply to the heaters during the experiment so that the fuel injected from the injector was vaporized instantly after impinging on the high temperature inner wall of the evaporator. The vaporized fuel and air were mixed in the evaporator and then supplied to the reactor through a baffle plate shown in Fig.1(b). This baffle plate was a 12mm-thick stainless steel disk with multiple holes drilled on it. Due to its multi-hole configuration, it was expected to enhance the mixing of fuel and air, and to prevent the flame from backfiring to the evaporator.

The reactor was made of a stainless steel pipe with inner diameter $D=$ $36.7 \mathrm{~mm}$ and $300 \mathrm{~mm}$ in length. Several ports were opened on the surface of the reactor. These ports could be used to insert probes for temperature measurement and gas sampling. Another electric band heater was twined around the reactor at the location of $3.1 \leq x / D \leq 4.3$. This heater was attached to preheat the reactor prior to the experiment so that the reaction starts smoothly once reactants are supplied to the reactor.

\subsection{Ceramic honeycomb}

In this fuel reformer, a cylindrical honeycomb ceramic of $34 \mathrm{~mm}$ in diameter and $40 \mathrm{~mm}$ in length was inserted in the reactor in order to obtain a wide range of reaction stability. The configuration and dimensions are shown in Fig. 1(c). This honeycomb was made of cordierite ceramic, having mesh of cell number $300 \mathrm{cpi}$ (square cells per square inch). A hydraulic diameter of a unit cell was $1.25 \mathrm{~mm}$ and the fraction of the open frontal area was $69 \%$.

\subsection{Measurement procedures}

The local gas temperature distribution was obtained by two types of thermocouple probes: radial-type and transversal-type. The radial-type probe was composed of two-holed ceramic tube (O.D. $3 \mathrm{~mm}$ ) and 0.1mm K-type thermocouple wires. The thermocouple wires were welded together at the ceramic tube tip. This probe was radially inserted into the reactor at each port located on the reactor side-wall so that the gas temperatures at multiple locations could be measured simultaneously during the experiment. The transversal-type probe, on the other hand, was composed of $0.1 \mathrm{~mm}$ K-type thermocouple wires inserted from the uppermost port of the evaporator and stretched along the center axis of the reformer. By using the transversal-type probe, it was possible to measure detailed gas temperature distributions along with the center axis of the reactor. All of the welded junctions of the thermocouple probes were coated with silica particles by which the probe was prevented from both deteriorating and working as a catalyst for the reaction. Signals from all the probes were recorded by a personal computer through a digital multi-thermometer (Keyence; NR1000). The sampling rate and accuracy of the temperature measurement was $1 \mathrm{~Hz}$ and $\pm 1 \mathrm{~K}$, respectively. 
Gas sampling for gas component analysis was conducted by inserting a sampling probe into the gas sampling port located at $x / D=7.08$. The sampling probe was made of stainless steel tube with $3 \mathrm{~mm}$ in outer diameter. The position of the tip end was set at the reactor centerline, and the reforming gas was collected by connecting the probe outlet to a one litter vacuum-collecting chamber. On the way to the vacuum bottle, sampling gas flew through a $0.2 \mathrm{~mm}$ hole nozzle which froze the gaseous reactions. The collected gas was then supplied to a gas chromatograph (Shimadzu; GC-8A) through a filtering chamber packed with silica gel, by which water and unburned methanol were removed from the gas. A gas component detector on the basis of TCD (Thermal Conductivity Detection) method was applied to the gas chromatograph. The column (Shinwa chem.; Shincarbon ST) mounted in the gas chromatograph oven was calibrated for $\mathrm{H}_{2}, \mathrm{~N}_{2}, \mathrm{O}_{2}, \mathrm{CO}$, and $\mathrm{CO}_{2}$ using external standard method. Argon was used as the carrier gas.

\subsection{Experimental conditions}

In this study, two cases of experiments were conducted. In Case 1, a ceramic honeycomb, called Honeycomb A was introduced at a single location $(1.74 \leq x / D \leq 2.83)$. Honeycomb A worked as a reaction stabilizer which avoided the reaction from blowoff or backfiring in wide range of thermal load, $q_{\text {load }}$. In Case 1,therefore, the thermal characteristic and the role of honeycomb ceramic in the fuel reformer were investigated. In Case 2, on the other hand, the secondary honeycomb called Honeycomb B was added in the downstream of Honeycomb, $4.47 \leq x / D \leq 5.56$. Honeycomb B worked as an adiabatic layer which increased the temperature level of the reaction and enhanced the reforming reaction. The adiabatic characteristic of Honeycomb B in terms of heat regeneration was discussed comparing the temperature profiles of Cases 1 and 2 .

In the present study, thermal and reaction characteristics of the methanol fuel reformer in terms of thermal load, $q_{\text {load, }}$, were investigated for both Cases 1 and 2.

Experiments were conducted at a fixed equivalence ratio $\phi=3.5$. The value of equivalence ratio is based on the complete combustion reaction. The stoichiometric equivalence ratio of POX, therefore, is $\phi_{\mathrm{st}}=3$. Table 1 shows the fuel and the air flow rates corresponding to each thermal load condition. The value of $q_{\text {load }}$ was determined by the fuel flow rate, $Q_{\text {fuel }}$, based on the lower heating value, $L H V$, of methanol.

Table 1: $\quad$ Fuel and air flow rate $(\phi=3.5)$.

\begin{tabular}{c|c|c|c}
\hline \hline$q_{\text {load }}[\mathrm{kW}]$ & $Q_{\text {fuel }}[\mathrm{mL} / \mathrm{min}]$ & $Q_{\text {air }}[\mathrm{L} / \mathrm{min}]$ & Ratio $*$ \\
\hline 0.37 & 1.40 & 1.65 & 1 \\
\hline 0.64 & 2.43 & 2.87 & 1.74 \\
\hline 1.04 & 4.00 & 4.71 & 2.85 \\
\hline 1.45 & 5.56 & 6.56 & 3.97 \\
\hline 1.76 & 6.73 & 7.94 & 4.81 \\
\hline 2.12 & 8.10 & 9.56 & 5.79 \\
\hline
\end{tabular}

*Flow rate ratio based on $q_{\text {load }}=0.37 \mathrm{~kW}$. 
The procedure applied in the experiment is described as follows. Prior to the experiment, the evaporator and the reactor were heated by the electric band heaters up to about $470 \mathrm{~K}$ and $820 \mathrm{~K}$, respectively. Then, the fuel and the air were supplied to the evaporator at the desired flow rates. After the reactants were supplied, the power supplied to the electric heaters was adjusted to keep the temperature inside the evaporator about $420 \mathrm{~K}$. On the other hand, the heaters attached to the reactor were turned off since the exothermic POX reaction sustains by itself in the reactor.

\section{Results and discussion}

\subsection{Temperature profiles}

In the present fuel reformer, a steady-state, super-rich flame of methanol could be stabilized due to the preheating and adiabatic characteristics of the ceramic honeycomb. As described in the previous section, the reactor was preheated up to $820 \mathrm{~K}$ prior to the experiment so that the premixed gas supplied was ignited by itself due to the high temperature environment in the reactor. Once the reaction started, the reaction was able to sustain by itself without the heat supply from the electric band heater in the reactor. In this section, the heat transfer characteristics in terms of temperature profiles within the reactor are discussed for understanding the thermal structure of the reformer.

Figure 2 shows the representative temperature profiles in Case 1 for several $q_{\text {load }}$ conditions varying from $0.37 \mathrm{~kW}$ to $2.12 \mathrm{~kW}$ at a constant $\phi=3.5$. A hatched region corresponds to Honeycomb $\mathrm{A}$, and a horizontal broken line indicates the calculated value of the adiabatic flame temperature, $T_{\mathrm{af}}$, based on the initial temperature condition of $420 \mathrm{~K}$ in the evaporator.

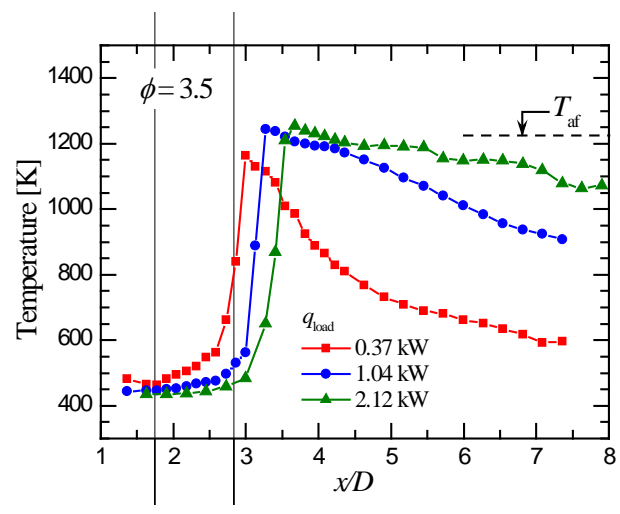

Figure 2: $\quad$ Temperature profiles (Case 1, $\phi=3.5$ ).

In all the cases, the reaction region was stabilized at the outlet of Honeycomb A, where the maximum temperature was observed. The value of the maximum temperature $T_{\max }$ reached close to $T_{\mathrm{af}}$, which indicates the reaction occurs almost 
in an adiabatic condition. The temperature in the downstream region of the reactor, $x / D>4.0$, on the other hand, increased with the increase of $q_{\text {load }}$. This is because the net heat amount of the reaction increased under larger $q_{\text {load }}$ conditions, lessening the effect of heat loss, and causing the temperature gradient at the downstream region of the reactor to become gentle in the larger $q_{\text {load }}$ conditions.

Figures 3(a) and (b) show the location of the maximum temperature, $(x / D)_{T \max }$, and the value of $T_{\max }$ with respect to $q_{\text {load }}$, respectively. In both figures, the square symbols correspond to the raw data obtained by the respective experiments, and the solid lines represent the averaged values of the experiments in the middle range conditions of $q_{\text {load }}, 0.64 \leq q_{\text {load }} \leq 1.76 \mathrm{~kW}$. The broken line in Fig. 3(b) shows the adiabatic flame temperature of $\phi=3.5$. As shown in Fig. 3(a), for the conditions of $0.64 \leq q_{\text {load }} \leq 1.76 \mathrm{~kW}$, the reaction region stayed at around $x / D=3.2$, indicating that the stable reaction occurred at an almostidentical location even in the wide range of $q_{\text {load }}$. The values of $T_{\max }$ corresponding to these $q_{\text {load }}$ conditions shown in Fig. $3(\mathrm{~b})$ stayed around $T_{\text {af }}$. In the smaller $q_{\text {load }}$ condition $\left(q_{\text {load }} \leq 0.64 \mathrm{~kW}\right)$, the value of $T_{\max }$ observed a slight decrease which is believed to be the effect of its relatively small reaction heat release rate.

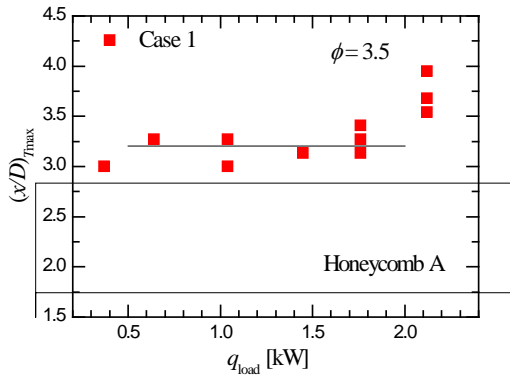

(a) The location of maximum temperature, $(x / D)_{T \max }$.

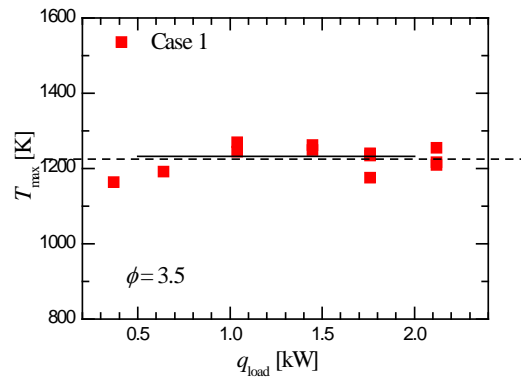

(b) Maximum measured temperature, $T_{\max }$.

Figure 3: Effects of $q_{\text {load}}$, on the reaction region and its temperature (Case 1, $\phi=3.5$ ).

In the higher $q_{\text {load }}$ conditions, on the other hand, the reaction location moved downstream, while the value of $T_{\max }$ did not change markedly. This phenomenon can be explained as "liftoff" of the reaction from Honeycomb A. The reaction region moved downstream due to its large mixture gas velocity, although the reaction itself kept on at the same level as for the stable conditions. Liftoff may cause unstable reaction within the reactor which is not suitable in a compact fuel reformer. It can also be estimated that "blowoff" of the reaction will occur when $q_{\text {load }}$ is increased beyond the stable limitation. 
Figures 4(a) and (b) show the locations of the maximum temperature and its values with respect to the $q_{\text {load }}$ conditions for both Cases 1 and 2. As shown in Fig. 4(a), the reaction region moved upstream by the effect of Honeycomb B in the cases of $q_{\text {load }}<1.45 \mathrm{~kW}$. For larger $q_{\text {load }}$ conditions, the effect of Honeycomb $\mathrm{B}$ on $(x / D)_{T \max }$ was not noticeable. Moreover, as the value of $q_{\text {load }}$ was decreased less than $0.64 \mathrm{~kW}$ in Case 2, the reaction region moved into Honeycomb A. This effect deteriorated the reaction efficiency, which will be discussed in the following section.

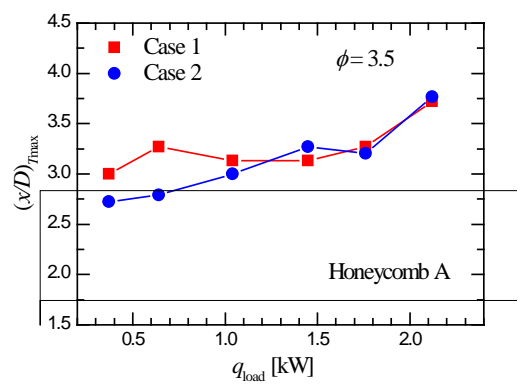

(a) The location of maximum temperature, $(x / D)_{T \max }$.

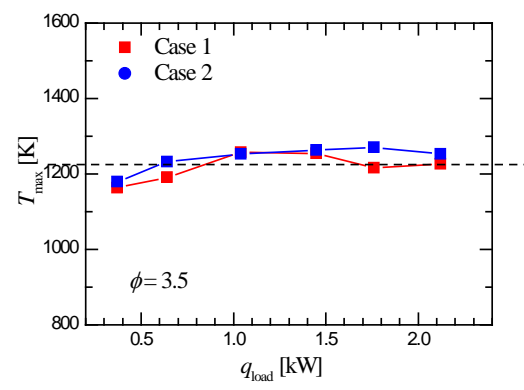

(b) Maximum measured temperature, $T_{\max }$.

Figure 4: Effects of secondary honeycomb on the reaction region and its temperature $(\phi=3.5)$.

Figure 4(b) shows the $T_{\max }$ distributions for Cases 1 and 2. Comparing with Case 1 , a slight increase of $T_{\max }$ was observed in most $q_{\text {load }}$ conditions for Case 2 . This temperature increase was caused by the energy regeneration brought by Honeycomb B. Honeycomb B absorbed a portion of enthalpy of the exhaust gas flowing through, and the solid surface of the honeycomb emitted radiation to the upstream region. By the rough calculation of the shape factor within the reactor, the radiation emitted from the upstream surface of Honeycomb B reaches to the reactor inner-wall surface by the ratio of $77 \%$, and the outlet surface of Honeycomb A by 23\%. Therefore, the energy regenerated from Honeycomb B was used mostly to warm up the reactor wall, which decreased the heat release to the wall from the exhaust gas. The remainder of the radiation contributed to warm up the vicinity of Honeycomb A and enhanced the preheating of the mixture gas. By both of these effects, the temperatures of the reaction and postreaction region were increased.

\subsection{Reforming characteristics}

Methanol conversion, $\alpha$, is defined in order to evaluate the efficiency of the present reformer:

$$
\alpha \equiv \frac{M_{\mathrm{CH} 3 \mathrm{OH}, \text { consumed }}}{M_{\mathrm{CH} 3 \mathrm{OH}, \text { supplied }}}=\frac{M_{\mathrm{CO}}+M_{\mathrm{CO} 2}}{M_{\mathrm{CH} 3 \mathrm{OH}, \text { supplied }}}=\frac{Y_{\mathrm{CO}}+Y_{\mathrm{CO} 2}}{Y_{\mathrm{N} 2}} \times \frac{M_{\mathrm{N} 2}}{M_{\mathrm{CH} 3 \mathrm{OH}, \text { supplied }}},
$$


where $M_{X}$ and $Y_{X}$ are the mole flow rate and the concentration of species $X$, respectively.

Figure 5 shows the dependence of $\alpha$ on $q_{\text {load }}$ for Cases 1 and 2. In Case 1 , the value of $\alpha$ did not change remarkably. It is estimated from this result that the reaction efficiency is not affected by $q_{\text {load }}$ condition as long as the reaction is stabilized at the outlet of Honeycomb A. For Case 2, on the other hand, $\alpha$ enhanced in conditions of $0.64 \leq q_{\text {load }} \leq 1.74 \mathrm{~kW}$. This enhancement was caused by the heat regeneration effect by Honeycomb B. Radiation emission from Honeycomb B caused a slight temperature rise of the reaction region (see Fig. 4(b)), and decrease of the heat loss through the reactor wall surface. By these effects, the methanol decomposition was enhanced and resulted in higher methanol conversion.

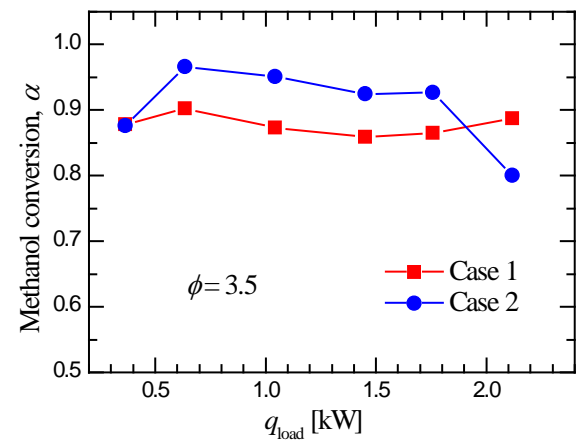

Figure 5: $\quad$ Dependence of $\alpha$ on $q_{\text {load }}$ (Cases 1 and 2).

In the condition of $q_{\text {load }}=0.37 \mathrm{~kW}$ in Case 2, the value of $\alpha$ decreased compared with $q_{\text {load }}=0.64 \mathrm{~kW}$. This deterioration of $\alpha$ was an effect of the reaction region moving into Honeycomb A. If a solid surface exists adjacent to the reaction region, the reaction heat is used to heat up not only the combustion gas, but also the solid phase of the honeycomb. This effect decreased the maximum temperature of the reaction region. Moreover, the high temperature solid surface emits radiation to the open space in the downstream region of the honeycomb, which causes an additional heat loss from the reaction region. By these effects, the temperature at the reaction region dropped and the reaction was deteriorated.

In the case of $q_{\text {load }}=2.12 \mathrm{~kW}$ for Case 2 , the value of $\alpha$ also decreased drastically. This was also caused by the existence of the solid phase of, in this case, Honeycomb B. In large $q_{\text {load }}$ conditions, the reaction region will liftoff and approaches to Honeycomb B. In this case Honeycomb B will quench the reaction and reduces the value of $\alpha$. From this effect, the radiation emitter should be placed in a certain distance from the reaction region in order to avoid undesired temperature drop, which deteriorate the reforming efficiency drastically. 


\section{Conclusions}

In the present study, the thermal and reaction characteristics of a several kilowatt-class compact methanol fuel reformer were investigated in terms of reaction location and temperature. The conclusions obtained from the discussion are summarized as follows:

- In the present conditions, the maximum measured temperature almost reached the adiabatic flame temperature in the downstream region close to the outlet surface of Honeycomb A.

- Stable reaction could be held at an almost-identical location at the outlet of Honeycomb A under a wide range of thermal loads. However, more increase of thermal load eventually caused "blowoff" of the reaction.

- In Case 2, the reaction region moved upstream and the temperature became slightly higher, which led to higher methanol conversion? This was caused by the energy regeneration effect by Honeycomb B.

- For the smallest and largest $q_{\text {load }}$ conditions in Case 2, the deterioration in methanol conversion was observed. This is due to the existence of the solid surface (i.e. ceramic honeycomb) within the reaction region.

\section{References}

[1] Demirbas, A. (2007) Progress and Recent Trends in Biofuels. Prog. Energy and Combust. Sci., 33, 1-18.

[2] Cantrell, K.B., Ducey, T., Ro, K.S., and Hunt, P.G. (2008) Livestock Wasteto-Bioenergy Generation Opportunities. Bioresour. Technol., 99, 79417953.

[3] Min, D.K., and Shin, H.D. (1991) Laminar Premixed Flame Stabilized inside a Honeycomb Ceramic. Int. J. Heat Mass Transf., 34(2), 341-356.

[4] Lammers, F.A., and Goey, L.P.H. (2003) A Numerical Study of Flash Back of Laminar Premixed Flames in Ceramic-Foam Surface Burners. Combust. Flame, 133, 47-61.

[5] Mendes, M.A.A., Pereira, J.M.C., and Pereira, J.C.F. (2008) A Numerical Study of the Stability of One-Dimensional Laminar Premixed Flames in Inert Porous Media. Combust. Flame, 153, 525-539.

[6] Echigo, R. (1982) Effective Conversion Method between Gas Enthalpy and Thermal Radiation and Its Application to Industrial Furnaces. Trans. Japan Soc. Mech. Eng., Ser. B (in Japanese), 4(435), 2315-2323.

[7] Okuyama, M., Echigo, R., Yoshida, H., Koda, M., and Hanamura, K. (1994) Spectral Radiation Properties of Super Fuel-Rich Premixed Flame. Trans. Japan Soc. Mech. Eng., Ser. B (in Japanese), 60(577), 3145-3152.

[8] Rai, Y., Tatsumi, K., and Nakabe, K. (2010) Experimental Study on a Compact Methanol-Fueled Reformer with Heat Regeneration Using Ceramic Honeycomb. Proc. Int. Heat Transf. Conf. 14, IHTC14-22742. 
[9] Rai, Y., Kogame, H., Tatsumi, K., and Nakabe, K. (2011) Experimental Study on a Compact Methanol-Fueled Reformer with Heat Regeneration Using Ceramic Honeycomb (2nd Report: Reaction Region Detection by a Positive Ion Current Probe). Proc. Int. Conf. on Power Eng. 2011, POWER2011-55377. 